Volume 10. No.1, January 2022

International Journal of Emerging Trends in Engineering Research

Available Online at http://www.warse.org/IJETER/static/pdf/file/ijeter031012022.pdf

https://doi.org/10.30534/ijeter/2022/031012022

\title{
A Survey of Traditional and Customized Bus Transportation Planning Methodologies
}

\author{
P.V.S. Anil Kumar ${ }^{1}$, Dr. Anuradha Purohit ${ }^{2}$ \\ ${ }^{1}$ M. E. Computer Engineering S.G.S.I.T.S Indore, India, pvsanil.kumar321@gmail.com \\ ${ }^{2}$ Associate Prof. Dept. of Computer Engineering S.G.S.I.T.S Indore, India, anuradhapurohit78@gmail.com
}

Received Date December 04, 2021 Accepted Date : December 30, 2021 Published Date : January 07, 2022

\begin{abstract}
Transportation planning is an area of public policy that is increasingly recognized for having a significant impact on human health and well-being. Passengers all across the world are choosing bus transit as one of the most cost-effective ways of transportation. The number of passengers who use this mode of transportation is steadily increasing. According to statistics, the bus was India's most popular mode of transportation in 2014. A bus was viewed as a mode of transportation by 66 percent of families in rural areas and 62 percent of households in urban areas. With increased demand, there is a concern about efficiently organizing this service. Because a lack of planning can generate major problems in the real world, such as traffic jams and high operating expenses, it is a source of concern for corporate and government entities who provide this service. In this paper, a review on various bus transit planning approaches and stages and methodologies used in each stage of the customized bus planning strategy is presented. This study will assist bus service organizing entities, whether private or public, in efficiently organizing bus service.
\end{abstract}

Key words: Clustering, DBSCAN, OD matrices, Smart cards.

\section{INTRODUCTION}

Bus organizational bodies are concerned about a substantial increase in the utilization of bus transportation services. This increased use leads to problems like increased traffic congestion, increased fuel consumption, which indirectly raises high operating costs, reduced community mobility etc. In light of the inefficient use of bus transit services and poor resource utilization, there is a pressing need to improve transportation system design. When seen broadly, bus planning encompasses aspects such as route planning, capacity expansion, ticket management, operations management, operator efficiency, customer orientation and the list goes on. Traffic congestion, air pollution, and traffic accidents can all be alleviated with the right organization of a bus transit service. Changes in the technique of travel and the quality of travel have been observed across time. Passengers have begun to express a need for a comfortable journey that meets their needs in terms of time. The statistics in Figure 1 represent that in Latin America, Asia, Europe, and Northern America regions the number of passengers traveling by bus daily is more as compared to the other regions. These statistics were taken from BRT data 2021 [1]. As an important part of diversified public transportation, the customized planning approach used in planning provides a customized medium-to-high-end public transportation service for people with the same or similar travel start and endpoints, travel time, and service needs, by keeping in mind, individual transportation needs [2]. Many researchers have worked on this topic. Some of them suggested the use of the traditional approach and others a customized approach. A customized approach has the advantage of shortening unnecessary waste of time and reduces the operating costs of customized buses [3].

The rest of the paper is organized as follows: background study of various approaches used in bus transit planning has been presented in section 2. Section 3, discusses the work done by researchers under different stages of the customized bus planning approach. Comparison of traditional and customized bus planning approach has been presented in section 4 . Finally, the conclusions are presented in section 5 .

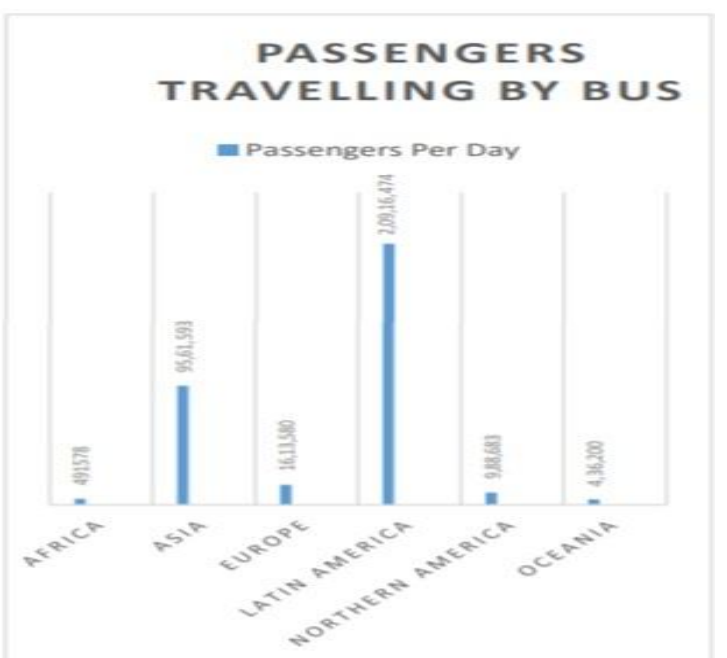

Figure 1: Number of Passengers travelling in various regions through bus 
P.V.S. Anil Kumar et al., International Journal of Emerging Trends in Engineering Research, 10(1), January 2022, 15 - 19

\section{BACKGROUND STUDY}

Various approaches used in bus transit planning is discussed in this section.

\subsection{Traditional Approach}

The traditional bus service planning system is used since the invention of the bus service. The primary motive of this service is to provide a relatively consistent service that runs on a fixed route and on a predecided timetable daily [4]. Buses running under this service cover all the stops in a preplanned route by reaching each station at a scheduled time throughout the day, irrespective of the presence of passengers at these stations. The advantage of using this service is its frequent availability daily. Traditional approach lacs in areas like service innovation, standards, transportation capacity loss when it comes to long-distance travel. If such a situation is neglected then it would raise concerns of high operational costs.

\subsection{Customized Approach}

Customized Bus Service planning came into use in the era of big data. The use of customized approach became more evident when researchers proved that the use of big data can cultivate a new reality in bus transport planning. The motive behind the introduction of this service is to cope with the diversified changes occurring in the lifestyles, time constraints, and quality and method of traveling being opted by passengers. The passenger communicates his pickup information using application, designed by specific bus service provider. Then a background analysis of the received pickup location is done and finally a decision is taken, when the request meets both the bus providers revenue requirements and the passenger travel time. Finally, the passenger gets the information regarding the service like pickup time on the same application. After the request is accepted by the provider, it departs a bus from the parking lot and carries the requested passenger from the residential area [5-6]. After servicing the passengers, the bus return to the parking lot. The above process is a single operation and it never exceeds the maximum passenger capacity of the vehicle. The disadvantages of traditional bus approach include a set position for a bus stop, a fixed line of vehicle operation, and a crowded environment.

\subsubsection{Steps Involved in Customized Bus Planning Approach}

Planning this service broadly consists of three stages [7]. They are as follows:

\section{A. Getting information of stations from the data sources}

In order to arrange a transit service for a zone, the foremost step is to have a knowledge of stations used by passengers in that zone. This information can be obtained from various sources. However, collection of this data has changed and evolved with time.

- Conducting surveys was the starting point of collecting information on stations. This was carried out by a team of people appointed by the bus service organizing body. These teams used to split into zones and from people present there, get information of stations used by them and when they used them. However, this wasn't a successful way because it consumed a lot of time and human effort. And had chances of human error.

- With the advancements in the use of technology, all this information of stations were collected by machine and this system was known as Automatic Fare Collection System (AFC's). As a coin has two faces AFC's also lacked in collecting information of destination stations of passengers. With the advancements in this field came the Smart Cards in the 1970s which looked similar to debit/credit cards and passengers have to swipe them at boarding as well as destination stations. Now as per the recorded distance between the two stations amount present in the card was deducted. The advantage of this card system is that it saves the data collection time and eliminates the chances of human error. Also, it has been perceived as a secure method of user validation and fare payment and has allowed an improved understanding of travel behavior patterns This is the reason why Smart cards are still used in the 2021's.

\section{B. Creating origin-destination matrix of bus stations}

This is an intermediate step of the approach where an origin-destination (OD) matrix is constructed from the information of stops. After getting the information of bus stops from the data sources, an origin-destination matrix will be constructed using some models that meet the requirement. Origin Destination(O-D) matrix is a two-D array. The origin-destination matrix is a matrix which is each cell represent the number of trips from origin (row) to the destination (column). The O-D matrix is important for transportation analysis, design, and management. It gives indispensable information on the travel demand between two different locations, which is used in many transportation applications from strategic planning to traffic control and management. Origin $\square$ destination $(\mathrm{O} \square \mathrm{D}$ ) provides a detailed picture of the trip patterns and travel choices of a cities or region's residents. Passengers' O-D matrices are used for service design purposes like This information is used for service planning and management to ensure adequate capacity, efficiency, customer service and cost reductions are 
P.V.S. Anil Kumar et al., International Journal of Emerging Trends in Engineering Research, 10(1), January 2022, 15 - 19

provided. Origin Destination (O-D) estimation, allowing a better understanding of individual travel patterns and improving strategic public transport planning.

\section{Estimation of OD Matrix}

The Origin-Destination matrix is usually estimated in two ways. They are:

- In conventional way, we can count the people trip from their origin to the destination by conducting a survey. But, conducting a survey would spend a lot of resources.

- Using synthetic models constructed from extant, autonomous software components whose existence and purpose are independent of the underlying model they comprise. Finally, the pair of origin-destination stops, where the number of trips is higher is chosen by the planning team, they usually name these selected stops as common stops to differentiate from the regular stops. Then these common stops are then passed on for the final step.

\section{$C$. Clustering the common stops selected from the origin destination matrix}

This is the final step of the approach where the clustering technique is applied on the common stops. As it is known that Automatic Fare collection data do not capture socioeconomic and trip purpose attributes. So, to overcome this limitation and improve the understanding of passenger behavior, some studies have focused on devising appropriate methodologies for the identification of activity patterns. Especially, the presence of longitudinal geospatial data has directed research attention to adopted clustering techniques to segmentation homogeneous groups of transit users and frequent travel patterns using the information of common stops. Finally, a clustering algorithm is applied on the identified common stops received from the intermediate step, to get varieties of information like identifying regular spatial and temporal patterns and understand the travel patterns of transit user. These results would help planners to find hidden behavior patterns and help them in scheduling line services so as to cover the demand variations adequately.

\section{LITERATURE REVIEW}

A lot of work done has been done by researchers in customized bus planning approach. The general way introduced by most of them includes three stages. They are getting information of stations from the data sources, creating an origin-destination (O-D) matrix of the bus stations and clustering the common stops selected from the origin-destination matrix. Literature review done by various researchers in each step of customized bus transit planning has been presented in this section.

\section{A. Work done in Smart card}

M. Bagchi et. al. [8] were the first ones who presented a review, on the nature of smart card data. What they found was that this data source can be helpful in bus travel planning. And also concluded that if this data source is taken into consideration, then it would create an impact on the data collection process and also impact on travel behavior analysis.

Jiang Ping Zhou et. al. [9] presented that smart card data's can be used to unveil the rhythm of daily commuting patterns as this data has a capacity to monitor and store observations for a long. They used Brisbane, Australia smart card which was used for more than half a year to derive origin destinations. And started monitoring in these areas. And found that there were variations every day and found that these variations lied in a consistent range and if taken into consideration could help in planning and outlier detection in commuting patterns.

\section{B. Work done in OD Matrices}

Di Huang, Jun Yu et. al. [10] introduced a framework for obtaining a stop-to-stop O-D trip information. This framework made collective use of automatic fare collection systems (AFC's) and GPS data of running buses to create an O-D matrix.

Azalden A. Alsger, Mahmoud Mesbah et. al. [11] presented a study on the consistency of the O-D matrix. In this study, they used to create an od matrix using different methodologies and finally compared the accuracy of each matrix formed using a different method. They found that the accuracy of the matrix was influenced by the use of multiple assumptions made for the calculation of alignment stops.

Tamara Dijukic et. al [12] introduced a new OD estimation methodology. This methodology makes use of eigenvectors and principal demand components as a fixed structure to create an od matrix instead of OD flows.

Cui et. al. [13], introduced an algorithm that estimates bus passenger trip origin-destination matrix using Chicago, US automatic fare collection data. This algorithm runs in three stages. In the first stage, data preparation is done. In the second stage single route origin-destination (OD) matrix for all routes were estimated using the iterative proportional fitting method and. Finally, in the last stage network-level, the origin-destination matrix was estimated from the transfer flows which they fetched out from consecutive transactions in automatic fare collection data (AFC).

\section{Work done in Clustering Algorithms}

T. N. Tran, K. Drab et. al. [14] introduced a concept of core density reachable chains for dealing with the issue of border points. As the name suggests this chain only includes core 
points on which clustering is performed. And after detection of all the clusters, the border points were assigned to closest density reachable chains, solving the problem of detection of border points of adjacent clusters.

K. Mahesh Kumar et. al. [15] introduced an approach to perform neighborhood search operation in the DBSCAN algorithm. They implemented this approach with the help of a graph-based index structure which is a graph-based implementation including vertices and edges instead of an Rtree index structure and observed that they got exactly the same results in a lesser computational time.

Animesh Tripathy, Sumit Kumar Maji et. al. [16] presented the use of concept reachability density instead of using the concept of core points, border points to perform clustering. And by doing this they achieved to perform the task of clustering with one parameter Minpts which is taken from the user end and made the other used parameter Eps adoptive.

Athanasios Salamanis et. al. [17] presented the use of the DBSCAN algorithm along with some predicting model in the field of traffic prediction. They performed a clustering operation on the traffic dataset and after that used some predicting models on separate clusters to understand the traffic patterns in each group so that abnormal situations like traffic jams can be predicted and required action can be taken.

L. M. Kieu, A. Bhaskar et. al. [18] introduced a Weighted stop density-based clustering algorithm (WS- 4 DBSCAN) for performing travel pattern analysis. This algorithm performs a two-level analysis process. In the first level of analysis, it makes use of existing knowledge of individual travel pattern to perform clustering. And in the second level, it performs neighbourhood search operation only when required. This way the algorithm showed great results in terms of complexity, by bringing the quadratic level complexity of DBSCAN to a subquadratic level in WS-DBSCAN.

L. M. Kieu, A. Bhaskar et. al. [19] presented the use of the DBSCAN algorithm to segment passengers into different classes. This research work was carried out on the demand of CCB operators. CCB operators were failing to provide to the point service to their passengers. So, the authors collected data from these operators and then segmented the passengers into four different classes namely transit passengers, regular passengers, regular passengers and irregular passengers. Using this information CCB operators were successful in providing specific services to specific classes of passengers.

\section{COMPARISON OF METHODS USED FOR BUS TRANSPORT PLANNING}

Traditional approach described in Section 2.1 have been used successfully in bus transit planning. Some of the advantages of using this approach are as given:

- In this approach bus follows a strict time table so availability of a bus is more frequent.

- As the bus runs on a fixed route, a passenger can catch a bus on the next stop by seeing the timetable of the bus, if he misses his bus.

Traditional approach is effective in bus transit planning but there are two main limitations which are identified. These limitations are as follows:

- In this approach bus transportation people are forced to adhere to strict timetables and fixed routes on their journeys.

- Chance of a bus going on a needless journey is higher as there is no way of knowing whether the trip is worthwhile or not until completion. Due to these limitations, researchers have focused on customized approach for bus transit planning. In this approach bus facilities are provided keeping in view passengers demand and request. Some of the advantages of this approach identified are as given:

- In terms of comfort, ride in a customized bus have higher comforts than a ride in traditional bus.

- The customized bus can attract commuters who take taxis, thereby reducing the use of cars and alleviating traffic congestion.

- A customized bus is equivalent to 8-9 cars, thereby it helps in utilizing road resources.

- Compared with a taxi, travelling cost is lower in bus.

\section{CONCLUSION}

In this paper comparative study of different approaches described by various researchers for planning a bus transit service has been presented. Lack of efficient transit planning problems leads to serious concerns like decrease in fuel efficiency resulting in high operational cost, improving road congestions, decrease of commuter productivity, etc. A lot of research has been done in redefining the planning approach so as to provide a better service. Various approaches used in the redefining process in each stage of planning is presented in this paper. 
P.V.S. Anil Kumar et al., International Journal of Emerging Trends in Engineering Research, 10(1), January 2022, 15 - 19

Our contribution in this paper can be summarized as follows:

- The theoretical concept of the traditional and customized bus planning approach has been presented.

- Various steps in a customized bus planning approach have been identified and discussed.

- Different methods used in each stage of the customized approach that make the planning process more efficient has been discussed.

\section{ACKNOWLEDGEMENT}

My thanks to Dr. Anuradha Purohit who have contributed towards the development of the paper.

\section{REFERENCES}

1. J. U. Duncombe, BRT+ Centre of Excellence, V. M. (n.d.). Global BRT Data. Retrieved from http://BRTData.org.

2. Hu YC, Chen X, Luo JL. (2018). Customized bus route planning model for multi-terminal and multi-model mixed load, J. Journal of Guangxi Normal University (Natural Science Edition), 36 (04): 1-11.

3. Guoling Lang (2020). A summary of research on customized bus route optimization for commuter needs, IOP Conf. Series: Earth and Environmental Science 546, doi:10.1088/1755-1315/546/5/052037.

4. Amita Johar. Planning and Operation of Urban Bus Route- A Case Study in West Zone of Delhi, January 2014 in ICETET Conference.

5. VUCHIC V R. (2005). Urban Transit: Operations, Planning and Economics, John Wiley \& Sons Publishing, New Jersey.

6. CEDER A. (2007). Public Transit Planning and Operation Theory, Modeling and Practice. Elsevier Publishing, Amsterdam.

7. Qiu, G., Song, R., He, S., Xu, W., Jiang, M., 2018. Clustering passenger trip data for the potential passenger investigation and line design of customized commuter bus, IEEE Trans. Intell. Transp. Syst. 2018, $1-10$.

8. M. Bagchi and P. R White. What role for smart-card data from bus systems, Proceedings of the institution of civil engineers-Municipal engineer, ISSN 0965-0903, E-ISSN 1751-7699, Volume 157 Issue 1, March 2004 pp. 39-46.

9. Zhou, J. Murphy. Day-to-day variation in excess commuting, An exploratory study of Brisbane, Australia.J. Transp. Geogr. 2019, 74, 223-232.

10. Di Huang, Jun Yu. A Method of Bus OD Matrix Estimation Using Multisource Data, Journal of Advance transportation 2020.
11. Azalden A. Alsger, Mahmoud Mesbah, Luis Ferreira, Hamid Safi. Use of Smart Card Fare Data to Estimate Public Transport Origin- Destination Matrix, Journal of the Transportation Research Board, No. 2535, Transportation Research Board, Washington, D.C., 2015, pp. 88-96, April 2019.

12. Tamara Dijukic. Efficient real time OD Matrix Estimation Based on Principal Component Analysis, 2012 15th International IEEE Conference on Intelligent Transportation.

13. Cui A (2006). Bus passenger origin-destination matrix estimation using automated data collection systems, Dissertation, Massachusetts Institute of Technology.

14. T. N. Tran, K. Drab, and M. Daszykowski. Revised DBSCAN algorithm to cluster data with dense adjacent clusters, Chemometrics Intell. Lab. Syst., vol. 120, pp. 92-96, Jan. 2013.

15. K.Mahesh Kumar, A.Rama Mohan Reddy. A fast DBSCAN clustering algorithm by accelerating neighbour searching using Groups method, Elsevier Pattern Recognization Vol 58, pp. 39-48, October 2016.

16. Animesh Tripathy, Sumit Kumar Maji, Prashanta Kumar Patra. FDCA: A Fast Density-Based Clustering Algorithm for Spatial Database System, International Conference on Computer \& Communication Technology, 2011.

17. Athanasios Salamanis, Giorgos Margaritis, Dionysios D. Kehagias, Georgios Matzoulas, Dimitrios Tzovaras. Identifying patterns under both normal and abnormal traffic conditions for short-term traffic prediction, Elsevier, Transportation Research Procedia, Volume 22, 2017, Pages 665-674.

18. L.M. Kieu, A. Bhaskar, and E. Chung. A modified density-based scanning algorithm with noise for spatial travel pattern analysis from smart card AFC data, Transp. Res. C, Emerg. Technol., vol. 58, pp. 193-207, Sep. 2015.

19. L. M. Kieu, A. Bhaskar, and E. Chung. Passenger segmentation using smart card data, IEEE Trans. Intell. Transp. Syst., vol. 16, no. 3, pp.1537-1548, Jun. 2015. 